\title{
Detecting diffuse axonal injury in rat brainstems by diffusion tensor imaging and AQP4 expression
}

\author{
Wenbin Zheng ${ }^{\mathrm{a},}{ }^{*}$, Chunlin $\mathrm{Ma}^{\mathrm{b}}$, Lingmei Konga ${ }^{\mathrm{a}}$, Xiran Chen ${ }^{\mathrm{a}}$ and Wenyao Fan ${ }^{\mathrm{a}}$ \\ ${ }^{a}$ Departments of Radiology, the Second Affiliated Hospital, Medical College of Shantou University, \\ Shantou, China \\ ${ }^{b}$ Department of Radiology, Kiang Wu Hospital, Macao
}

\begin{abstract}
This study investigated the correlation between AQP4 expression and DTI in rat brainstems after diffuse axonal injuries (DAI). Forty rats were imaged before injury and reimaged at 3, 6, 12, 24 and $72 \mathrm{~h}$ post-injury. A control group of 8 rats was imaged and sacrificed for histology but not injured. After brain injury, AQP4 expression and ADC values in the brainstems increased gradually, reaching peak values at $24 \mathrm{~h}$ and $12 \mathrm{~h}$, respectively. FA values decreased within $72 \mathrm{~h}$. There was a negative correlation between ADC values and brainstem AQP4 expression at $12 \mathrm{~h}$, and a positive correlation at $24 \mathrm{~h}$ or $72 \mathrm{~h}(P<0.01)$, respectively. Changes in the ADC and FA values in the brainstems indicated brain edema and severe axonal injuries. The correlations between AQP4 expression and time-dependent ADC values aid in understanding brain edema development after DAI.
\end{abstract}

Keywords: Diffusion axonal injury, brain edema, AQP4, diffusion tensor imaging, apparent diffusion coefficient (ADC), fractional anisotropy (FA)

\section{Introduction}

In a series of experiments on human test subjects proved that the major component contributing to traumatic brain swelling and increased intracranial pressure (ICP) was not vascular engorgement, but rather a decreased CBF [1]. Thus, swelling and increased ICP relates to diffuse cytotoxic brain edema. Aquaporin-4 (AQP4) is a water-channel protein expressed strongly in the brain, predominantly in astrocyte endfeet membranes facing the blood-brain and brain-cerebrospinal fluid interfaces. Experiments using AQP4-null mice provide strong evidence for AQP4 involvement in cerebral water balance. In cytotoxic edema, AQP4 deletion slows the rate of water entry into the brain. In vasogenic edema, AQP4 deletion reduced the rate of water outflow from brain parenchyma [2-4].

Diffusion tensor MR imaging (DTI) can provide information about brain microstructures by quantifying isotropic and anisotropic water diffusion in detecting axonal injuries. Experimental evidence has shown that water diffusion has a directional asymmetry (anisotropy) in organized tissues,

\footnotetext{
* Address for correspondence: Wenbin Zheng, Departments of Radiology, the Second Affiliated Hospital, Medical College of Shantou University, Shantou, China. Tel.: 86075488915717; Fax: 86075488993282; E-mail: hwenb@126.com.
}

0959-2989/15/\$35.00 @ 2015 - IOS Press and the authors. 
such as muscles or brain white matter [5-12]. In white matter tracts, where most or all of the axons align in parallel, diffusion parallel to the axons is greater than diffusion perpendicular to the axons. Two DTI parameters, axial diffusivity and relative anisotropy, reflect the brain's structural integrity and the information of brain edema, and could represent the pathology change of the brain edema and axon injury post-DAI in vivo. Among biophysical mechanisms contributing to ADC changes, membrane permeability to water could play a substantial role [13]. In this study, we investigate the relationship between the AOP4 expression and ADC changes according to DAI pathological conditions.

\section{Materials and methods}

\subsection{Animal model}

Forty-eight male Sprague-Dawley rats, (weighing 280-320 g), were maintained under standard laboratory conditions with 12-hour light/dark cycles. Rats were treated in compliance with NIH guidelines for the care and use of laboratory animals. After one week of stabilization on an ad lib diet, rats were randomly assigned to five DAI groups and one control group. Rats were anesthetized with an intraperitoneal chloral hydrate injection $(0.3 \mathrm{ml} / \mathrm{kg}$ i.p.). A total of 40 anesthetized adult rats were injured using the impact-acceleration model of Marmarou [1]. To deliver DAI, animals were placed on a $20 \mathrm{~cm}$ thick sponge bed in a prone position. The injury was then induced by dropping a weight of $500 \mathrm{~g}$ from a height of $1.8 \mathrm{~m}$ onto the steel disk. Rebound impact was prevented by sliding the flexible sponge bed from the tube immediately. Following termination of the procedure, a plastic skull cap was secured over the impact site, the skin incision was sutured closed, and rats were returned to their normal environment and were provided with food and water. DAI rats were sacrificed at five different time points $(3,6,12,24$, and $72 \mathrm{~h})$ after imaging post injury. A control group of 8 rats was imaged and sacrificed for histology, but not injured. All procedures complied with the Shantou University Guide for Care and Use of Laboratory animals, and were approved by the Shantou University Medical College Animal Use Committee.

\subsection{Imaging}

Conventional MRI and DTI were performed in all rats. The body temperatures of all rats were maintained throughout MRI acquisition. Five separate parallel groups of 8 rats per experimental group (DAI group) were imaged at 3, 6, 12, 24, and $72 \mathrm{~h}$ after injury. Images were obtained using a 1.5TMR imaging system (GE Signa) equipped with high performance gradients. MRI parameters were as follows: T1 weighted images (T1WI) were obtained using TR (repetition period)/spin-echo time $(\mathrm{TE})=1290 \mathrm{~ms} / 23.2 \mathrm{~ms}, \mathrm{NEX}=2$, section thickness $=3 \mathrm{~mm}$, matrix $=256 \times 256$, and field of view $($ FOV $)=12 \mathrm{~cm} \times 12 \mathrm{~cm}$. T2 weighted images $(\mathrm{T} 2 \mathrm{WI})$ were obtained using a fast spin echo sequence, $\mathrm{TR} / \mathrm{TE}=4420 \mathrm{~ms} / 107.9 \mathrm{~ms}, \mathrm{NEX}=2$, section thickness $=3 \mathrm{~mm}$, matrix $=256 \times 256$, FOV $=12 \mathrm{~cm} \times 12 \mathrm{~cm}$. TSE factor=10, DTI was obtained with a single-shot echo planar imaging (EPI) sequence by using 25 diffusion-encoding directions, TR/TE $=6000 \mathrm{~ms} / 107.7 \mathrm{~ms}$, NEX $=2$, section thickness $=3 \mathrm{~mm}$, spacing $=0$, matrix $=128 \times 128$. Display field of view $($ DFOV $)=6 \mathrm{~cm} \times 6 \mathrm{~cm}$, and the $b$ value was $1000 \mathrm{~s} / \mathrm{mm}^{2}$.

\subsection{Data processing}


Images were post-processed offline using DTI postprocessed software (Functool 4.5.5 software) of an Advantage workstation for Windows (AW4.3, GE Healthcare). After correction for movement and EPI-induced distortion artifacts by Functool 4.5.5 software, the diffusion tensor was calculated for each voxel, which automatically computed the FA and ADC maps. The region of interest was about 5 $\mathrm{mm}^{2}$ and was traced on the brainstem in the original DTI transverse slice image to avoid influence from subjective factors. The average value of FA and ADC values were measured by two experienced radiologists blinded to the animals' statuses.

\subsection{Histology}

Rats were sacrificed for histological examination immediately following MRI exams. Animals were overdosed with chloral hydrate intraperitoneally, transcardially perfused with $0.1 \mathrm{M}$ phosphate-buffered saline (PBS). Brains were extracted from the skull, and then fixed with $4 \%$ paraformaldehyde (PFA) in $0.1 \mathrm{M}$ phosphate buffer $(300-400 \mathrm{ml})$ for $24 \mathrm{~h}$. The brains were then embedded in paraffin, and contiguous $5 \mu \mathrm{m}$ sections at the brainstem were cut on a microtome (Rm 2016, LEICA, Germany). Sections were stained with hematoxylin and eosin (HE) or Bielschowsky's silver stain.

\subsection{Immunostaining}

The sections were being washed with PBS, and then treated with $0.3 \%$ hydrogen peroxide for 10 min to deactivate endogenous peroxidase. After being washed 3 times, 5 min each, with $0.01 \mathrm{M} \mathrm{PBS}$, sections were blocked in $10 \%$ goat serum for $10 \mathrm{~min}$ at room temperature. Then sections were incubated with ready to use rabbit anti-AQP4 (BA-1560, Wuhan, China) overnight at $4^{\circ} \mathrm{C}$, followed by a $30 \mathrm{~min}$ incubation at $37^{\circ} \mathrm{C}$ with biotinylated goat anti-rabbit secondary antibody (ZDR-5306, Beijing, China).

Image acquisition was performed using an Olympus digital camera and dedicated software. AQP4 quantification was using an Image-Pro Plus 6.0 microimage analysis system.

\subsection{Statistical analysis}

Statistical analyses were performed using SPSS19.0 software (SPSS, Chicago, IL, USA). ADC and FA values and AQP4 expression were reported as the mean \pm standard deviation $(\mathrm{X} \pm \mathrm{SD})$ in each group. For comparisons within each group and between groups, we used Student's t-test. Correlations between DTI parameters and AQP4 expression were calculated using the Pearson test. A $P<0.05$ value was considered significant.

\section{Results}

\subsection{Conventional MRI results}

T1WI and T2WI maps showed clear brain parenchyma structure. There were no difference in signal intensity between the control group and DAI group.

\subsection{DTI imaging}




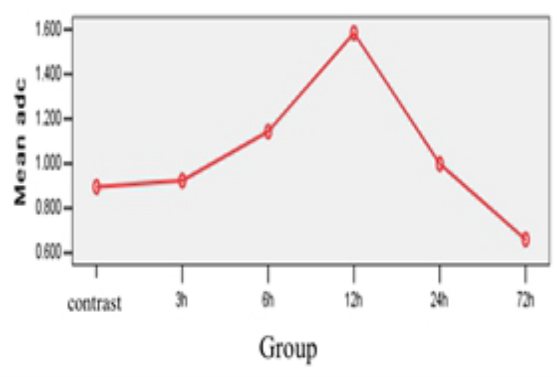

Fig. 1. ADC value of control group and DAI groups.

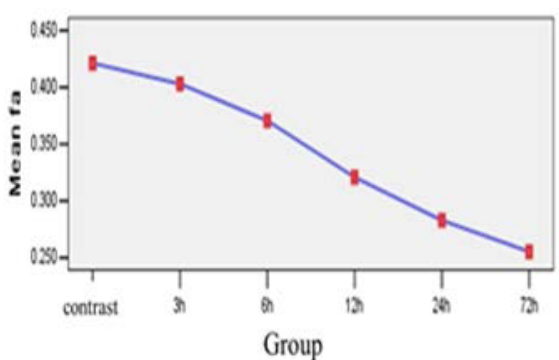

Fig. 2. FA value of control group and DAI groups.

The ADC value in the control group was $0.896 \pm 0.121 \times 10^{-3} \mathrm{~mm}^{2} / \mathrm{s}$. In the DAI group, ADC values showed a slight increase at $3 \mathrm{~h}$, and an obvious increase at 3-12 $\mathrm{h}$; they reached a peak $(1.584 \pm 0.200$ $\left.\times 10^{-3} \mathrm{~mm}^{2} / \mathrm{s}\right)$ at $12 \mathrm{~h}$, and then decreased. The ADC decreased obviously at $72 \mathrm{~h}(0.660 \pm 0.127-3$ $\mathrm{mm}^{2} / \mathrm{s}$, Figure 1), which was lower than that in the control group. An LSD-t test was run at each time point compared to the control group sample, which was considered significant $(P<0.05)$ at $6 \mathrm{~h}$ and 12 $\mathrm{h}$ post-DAI, and obviously significant $(P<0.01)$ at $72 \mathrm{~h}$. The FA value in the control group was $0.421 \pm 0.006$. In the DAI group, it continually decreased and reached a minimum value $(0.255 \pm 0.005)$ at $24 \mathrm{~h}$ after injury (Figure 2). An LSD-t test was run at every time point compared to the control group sample, which was considered obviously significant $(P<0.01)$.

\subsection{Histological}

\subsection{1. $H E$}

No pathologic changes in the brainstem were detected in the control rats. The DAI group showed expanded cells, expanded extracellular cell space, weakly stained plasma, enlarged axon space, axonal retraction bulbs, and shrunken endotheliums.

\subsubsection{Bielschowsky's silver stain}

The axons of silver stain were well distributed and regularly arranged without being twisted or disrupted in control rats. In the DAI group, axons were swollen, twisted, and partly disorganized; generated axonal retraction bulbs were observed. There were many axonal retraction bulbs at $3 \mathrm{~h}$ post-injury; they increased obviously at 24-72 $\mathrm{h}$.

\subsubsection{AQP4 expression}

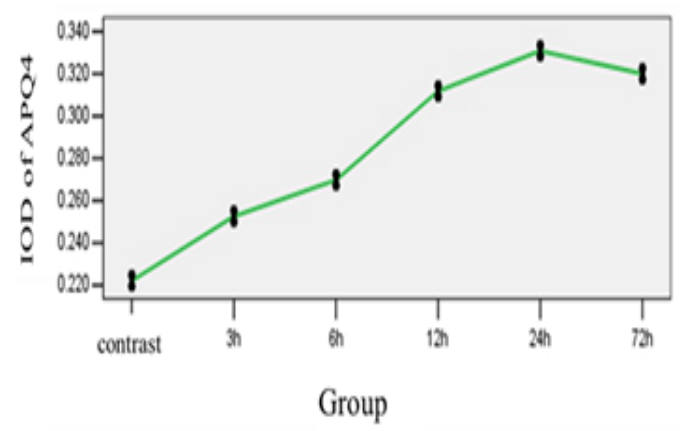

Fig. 3. AQP4 expression of control group and DAI groups. 
In the control group, the IOD of brainstem AQP4 was $0.228 \pm 0.021$. In the DAI group (Figure 3), the AQP4 immunoreactivity was strongly upregulated compared to the control group, reaching a peak at $24 \mathrm{~h}(0.369 \pm 0.028, P<0.01)$.

\subsection{Correlation between DTI imaging and AQP4 expression}

In the DAI groups, there was a significant positive correlation between the AQP4 expression with $\mathrm{ADC}$ values in $12 \mathrm{~h}$ and $72 \mathrm{~h}(r=0.691$ and $r=0.608 ; \mathrm{P}<0.01)$, and no correlation at $24 \mathrm{~h}(P>0.05)$. In $72 \mathrm{~h}$ after injury, the negative correlation of the AQP4 expression with FA values was significant $(r=-0.946 ; P<0.01)$. Besides, there was no correlation between ADC and FA values in $72 \mathrm{~h}$ after DAI $(P>0.05)$.

\section{Discussion}

AQP4 is expressed in glial cells at the blood-brain and the brain-CSF interfaces, which play an important role in maintaining brain water homeostasis [3]. Research has confirmed that increased AQP4 expression is implicated in brain edema $[14,15]$. Animal research regarding acute intoxication brain edema found that AQP4 expression increases in the brain parenchyma from the model, which means AQP4 plays an important role in brain edema formation [16]. The injury mechanism of DAI is complicated. Following TBI, various mediators are released, which enhance vasogenic and/or cytotoxic brain edema. The axon injury is not the primary change caused by external force, but the step-change from brain ischemia and edema [17]. The development of a neural axon injury is implicated in the secondary change caused by brain edema. The expression of AQP4 protein after DAI is time-dependent and possibly implicated in the formation and resolution of DAI-induced cerebral edema.

The histological feature in our study by using Marmarou's DAI model, showing that there is obviously edema in the brain stem $3 \mathrm{~h}$ post-injury, reaching a peak in 24-72 $\mathrm{h}$. In this study, it was discovered that the AQP4 expression of brainstems increased first, then decreased after DAI, which may be implicated in the type, level, and time of brain edema. Thus, we sought to establish that MRI correlates to traumatic axonal injury in an animal model where direct histological evaluation could be performed. When axonal injury occurs, the ADC temporarily increases in 40-60 minutes post-injury, then decreases and reaches to nadir at 7-14 $\mathrm{d}$ in the severe DAI model of rats [18]. The FA value decreases with tissue destruction because the FA value mainly reflects tissue integrality. The decreased degree of FA post-DAI relates to the axonal injury degree [19]. Our observation of decreased FA provides evidence of brainstem axonal injuries after DAI, consistent with prior studies using DTI to show reduced FA following TBI $[7,19]$. We hypothesized that water movement through AQP4 could affect the apparent diffusion coefficient (ADC) and fractional anisotropy (FA) measurements. Decreased ADC values reflect cytotoxic edema, which may be due to increase AQP4 expression; increased ADC values reflect vasogenic edema. We found a significant increase of ADC and AQP4 expression at $12 \mathrm{~h}$ post-DAI. We believe that the direct injury from the external force leads to the destruction of BBB and vasogenic edema, which takes the principal place. This injury increases extracellular fluid, brain extracellular space enlargement, and increases water diffusion. The ADC increased and FA decreased at the time. The vasogenic edema continued to exist and the AQP4 expression increases at $12 \mathrm{~h}$ or $24 \mathrm{~h}$ post-DAI in our study. On one hand, the AQP4 cleared the intercellular fluid to reabsorb fluid to BBB by transcellular transport. On the other hand, part of the 
water went into the cell caused by the AQP4, which facilitates the formation of cytotoxic brain edema. The proportion of cytotoxic brain edema began to increase, causing an ADC decrease. For the influence of vasogenic and cytotoxic brain edema, there is no statistical significance between AQP4 expression and ADC in this stage. The vasogenic edema released and the AQP4 up-regulation decreased $24 \mathrm{~h}$ and $72 \mathrm{~h}$ after injury. The organism down-regulates the AQP4 to release the facilitation of AQP4. The continued effect of vasogenic and cytotoxic edema decreased AQP4 and ADC.

\section{Conclusion}

Consistent with DTI predictions, there was histological detection of axonal injuries in the brain stem. ADC measurements in the brainstem correlated with AQP4 after DAI. Changes in AQP4 expression and time-dependent ADC values were helpful for reflecting the classification between vasogenic brain edema and cytotoxic brain edema. This research provides guidance for clinical treatment for different types of brain edema.

\section{Acknowledgment}

This work was supported by the Natural Science Foundation of Guangdong Province, China (Grant no. 2014A030313481), the Science and Technology Planning Project of Shantou, China (Grant no.201424260), and was sponsored by Shantou University Medical College Clinical Research Enhancement Initiative, China.

\section{References}

[1] A. Marmarou, M.A. Abd-Elfattah Foda, W. van den Brink, J. Campbell, H. Kita and K. Demetriadou, A new model of diffuse brain injury in rats, Part I: Pathophysiology and biomechanics, Journal of Neurosurgery 80 (1994), 291-300.

[2] P. Agre, L.S. King, M. Yasui, W.B. Guggino, O.P. Ottersen, Y. Fujiyoshi, A. Engel and S. Nielsen, Aquaporin water channels-From atomic structure to clinical medicine, Journal of Physiology 542 (2002), 3-16.

[3] J. Badaut, F. Lasbenes and P.J. MagistreRi, Aquaporins in brain: Distributions, physiology, and pathophysiology, International Society of Cerebral Blood Flow and Metabolism 22 (2002), 367-378.

[4] S. Nielsen, E.A. Nagelhus, M. Amiry-Moghaddam, C. Bourque, P. Agre and O.P. Ottersen, Specialized membrane domains for water transport in glial cells: High-resolution immunogold cytochemistry of aquaporin-4 in rat brain, Journal of Neuroscience 17 (1997), 171-180.

[5] K. Arfanakis, V.M. Haughton, J.D. Carew, B.P. Rogers, R.J. Dempsey and M.E. Meyerand, Diffusion tensor MR imaging in diffuse axonal injury, American Journal of Neuroradiology 23 (2002), 794-802.

[6] T.A. Huisman, L.H. Schwamm, P.W. Schaefer, W.J. Koroshetz, N. Shetty-Alva, Y. Ozsunar, O. Wu and A.G. Sorensen, Diffusion tensor imaging as potential biomarker of white matter injury in diffuse axonal injury, American Journal of Neuroradiology 25 (2004), 370-376.

[7] M. Inglese, S. Makani, G. Johnson, B.A. Cohen, J.A. Silver, O. Gonen and R.I. Grossman, Diffuse axonal injury in mild traumatic brain injury: A diffusion tensor imaging study, Journal of Neuroradiology 103 (2005), 298-303.

[8] S. Mori and J. Zhang, Principles of diffusion tensor imaging and its applications to basic neuroscience research, Neuron 51 (2006), 527-539.

[9] N. Nakayama, A. Okumura, J. Shinoda, Y.T. Yasokawa, K. Miwa, S.I. Yoshimura and T. Iwama, Evidence for white matter disruption in traumatic brain injury without macroscopic lesions, Journal of Neurology Neurosurgery Psychiatry 77 (2006), 850-855.

[10] P.W. Schaefer, P.E. Grant and R.G. Gonzalez, Diffusion-weighted MR imaging of the brain, Radiology 217 
(2000), 331-345.

[11] D.R. Rutgers, P. Fillard and G. Paradot, Diffusion tensor imaging characteristics of the corpus callosum in mild, moderate, and severe traumatic brain injury, American Journal of Neuroradiology 29 (2008), 1730-1735.

[12] V.F. Newcombe, G.B. Williams and J. Nortje, Analysis of acute traumatic axonal injury using diffusion tensor imaging, British Journal of Neurosurgery 21 (2007), 340-348.

[13] C.L. Chin, F.W. Wehrli, Y. Fan, S.N. Hwang, E.D. Schwartz, J. Nissanov and D.B. Hackney, Assessment of axonal fiber tract architecture in excised rat spinal cord by localized NMR q-space imaging: Simulations and experimental studies, Magnetic Resonance in Medicine 52 (2004), 733-740.

[14] M. Amiry-Moghaddam, T. Otsuka and P.D. Hurn, Analpha-syntrophin-dependent pool of AQP4 in astroglial end-feet confers bidirectional water flow between blood and brain, Proceedings of the National Academy of Sciences 100 (2003), 2106-2111.

[15] H. Hu, H.T. Yao and W.P. Zhang, Increased expression of aquaporin-4 in human traumatic brain injury and brain tumors, Journal of Zhejiang University (Science B) 6 (2005), 33-37.

[16] G. Manley, M. Fujimura and L. Ma, Aquaporin-4 deletion in mice reduces brain edema after acute water intoxication and ischemic stroke, Natural Medicine 6 (2000), 159-163.

[17] J.R. Stone, R.H. Singleton and J. Povlishock, Intra-axonal nerurofilament compaction dose not evoke local axonal swelling in all traumatically injured axons, Experimental Neurology 172 (2001), 320-331.

[18] P. Barzo, A. Marmarou, P. Fatouros, K. Hayasaki and F. Corwin, Contribution of vasogenic and cellular edema to traumatic brain swelling measured by diffusion-weighted imaging, Journal of Neurosurgery 87 (1997), 900-907.

[19] D. Ducreux, I. Huynh, P. Fillard, et al., Brain MR diffusion tensor imaging and fibre tracking to differentiate between two diffuse axonal injuries, Neuroradiology 47 (2005), 604-608. 